\title{
Sevoflurane post-conditioning reduces rat myocardial ischemia reperfusion injury through an increase in NOS and a decrease in phopshorylated NHE1 levels
}

\author{
JIANFANG CAO*, HONG XIE*, YING SUN, JIANG ZHU, MING YING, \\ SHIGANG QIAO, QIN SHAO, HAORONG WU ${ }^{* *}$ and CHEN WANG ${ }^{* *}$ \\ Department of Anesthesiology, The Second Affiliated Hospital of Soochow University, Suzhou, Jiangsu 215004, P.R. China
}

Received March 2, 2015; Accepted September 24, 2015

DOI: $10.3892 / \mathrm{ijmm} .2015 .2366$

\begin{abstract}
The protectiveeffects of sevofluranepost-conditioning against myocardial ischemia/reperfusion (I/R) injury (MIRI) have been previously reported. However, the mechanisms responsible for these protective effects remain elusive. In this study, in order to investigate the molecular mechanisms responsible for the protective effects of sevoflurane post-conditioning on isolated rat hearts subjected to MIRI, Sprague-Dawley rat hearts were randomly divided into the following 6 groups: i) the sham-operated control; ii) $2.5 \%$ sevoflurane; iii) ischemia/reperfusion (I/R); iv) $2.5 \%$ sevoflurane post-conditioning plus $\mathrm{I} / \mathrm{R}$; v) $2.5 \%$ sevoflurane post-conditioning + NG-nitro-Larginine methyl ester (L-NAME) plus I/R; and vi) L-NAME plus $I / R$. The infarct size was measured using 2,3,5-triphenyl tetrazolium chloride (TTC) staining. Additionally, the myocardial nitric oxide (NO), NO synthase (NOS) and nicotinamide adenine dinucleotide $\left(\mathrm{NAD}^{+}\right)$levels were determined. Autophagosomes and apoptosomes in the myocardium were detected by transmission electron microscopy. The levels of Bcl-2, cleaved caspase-3, Beclin-1, microtubule-associated protein light chain 3 (LC3)-I/II, $\mathrm{Na}^{+} / \mathrm{H}^{+}$exchanger 1 (NHE1) and phosphorylated NHE1 protein were measured by western blot analysis. NHE1 mRNA levels were measured by reverse transcription-quantitative polymerase chain reaction. Compared with the I/R group, 15 min of exposure to $2.5 \%$ sevoflurane during early reperfusion significantly decreased the myocardial infarct size, the autophagic vacuole numbers, the NHE1 mRNA and protein expression of cleaved caspase-3, Beclin-1 and LC3-I/II. Post-conditioning with $2.5 \%$ sevoflurane also increased the NO and NOS levels and Bcl-2 protein expres-
\end{abstract}

Correspondence to: Dr Chen Wang or Dr Haorong Wu, Department of Anesthesiology, The Second Affiliated Hospital of Soochow University, 1055 Sanxiang Road, Suzhou, Jiangsu 215004, P.R. China E-mail: wangchen1791@163.com

E-mail: 455261209@qq.com

**** Contributed equally

Key words: myocardial ischemia/reperfusion, sevoflurane postconditioning, apoptosis, autophagy, NOS/p-NHE1 sion $(\mathrm{P}<0.05$ or $\mathrm{P}<0.01)$. Notably, the cardioprotective effects of sevoflurane were partly abolished by the NOS inhibitor, L-NAME. The findings of the present study suggest that sevoflurane post-conditioning protects the myocardium against I/R injury and reduces the myocardial infarct size. The underlying protective mechanisms are associated with the inhibition of mitochondrial permeability transition pore opening, and with the attenuation of cardiomyoctye apoptosis and excessive autophagy. These effects are mediated through an increase in NOS and a decrease in phopshorylated NHE1 levels.

\section{Introduction}

Myocardial ischemia/reperfusion (I/R) injury (MIRI) causes irreversible damage to myocardial cells, decreases cell viability and may have life-threatening consequences (1). Despite the significant negative impact of MIRI on human health, alleviating $\mathrm{I} / \mathrm{R}$ injury remains a challenge for researchers and clinicians.

Previous studies have reported that ischemic post-conditioning, including pharmacological post-conditioning (2), may prevent $\mathrm{I} / \mathrm{R}$ injury in clinical practice. Ischemic postconditioning has several clinical advantages, including predictability, controllability and safety (3). It has recently been reported that inhalation anesthetics administered at the beginning of reperfusion provides cardioprotection against I/R injury (4). Sevoflurane is widely used in cardiac surgery since it has the advantages of shorter induction, reduced recovery time and higher safety when compared with other anesthetics (5). It has been demonstrated that sevoflurane reduces myocardial infarct size and mortality in both animals $(6,7)$ and humans (8). It has been suggested that a variety of molecular mechanisms may be responsible for the protective effects of sevoflurane, including phosphatidylinositol 3-kinase (PI3K)/Akt pathway activation $(4,9)$, reactive oxygen species (ROS) regulation (10) and mitochondrial permeability transition pore (MPTP) inhibition (11). However, the exact mechanisms through which sevoflurane post-conditioning reduces MIRI remain unknown.

ROS play an important role in MIRI (12), and they have been reported to modulate the activity of MPTPs (13). The MPTP is a multiprotein megachannel, and its opening can cause permeability transition. Massive MPTP opening can result in mitochondrial depolarization and lead to apoptosis or 
autophagy (14). Recent studies have demonstrated that nitric oxide (NO) inhibits sarcolemmal $\mathrm{Na}^{+} / \mathrm{H}^{+}$exchanger 1 (NHE1) activity by activating the soluble isoform of guanylyl cyclase (sGC) and cGMP-dependent protein kinase (PKG) (15). Both NO synthase (NOS) and NO have been reported to exert anti-apoptotic effects (16) and play important roles in the pathogenesis of MIRI (17).

NHE1 has been demonstrated to be a key factor protecting against I/R injury in experimental animal models (18). NHE1 is a major $\mathrm{Na}^{+}$influx pathway that couples $\mathrm{H}^{+}$efflux to $\mathrm{Na}^{+}$influx in a 1:1 ratio under the driving force of a $\mathrm{Na}^{+}$gradient formed by the $\mathrm{Na}^{+}$pump. NHE1 activation leads to elevated intracellular $\mathrm{Na}^{+}$concentrations and cytoplasmic alkalinization (19). Villa-Abrille et al (20) suggested that silencing cardiac mitochondrial NHE1 prevents MPTP opening.

Even though a number of studies have demonstrated the cardioprotective effects of NHE1 inhibitors against MIRI, it is unknown whether NOS and/or phosphorylated (p-)NHE1 plays a role in mediating the cardioprotective effects of sevoflurane post-conditioning. In the present study, we investigated the underlying mechanisms through which sevoflurane postconditioning reduces myocardial infarct size and mortality following MIRI. Using a rat heart model of $\mathrm{I} / \mathrm{R}$, we investigated new strategies for the basic and clinical treatment of MIRI.

\section{Materials and methods}

Animals and reagents. Adult male Sprague-Dawley rats weighing 270-350 g (9-10 weeks old; $n=144)$ were purchased from the Animal Center of Soochow University (Suzhou, China). Male rats were used to avoid any potentially confounding effects of female sex hormones (i.e., estrogen) on sevoflurane post-conditioning. All rats were kept under a 12-h light-dark cycle in a temperaturecontrolled environment, and were allowed free access to food and water for 1 week prior to euthanasia. All rats were randomly divided into the experimental groups described below following euthanasia (all animals were anaesthetized by single-dose intraperitoneal injection of pentobarbital, $50 \mathrm{mg} / \mathrm{kg}$ body weight). All experimental protocols were conducted in accordance with the Guidelines for the Care and Use of Laboratory Animals and the policies of Soochow University (protocol number: SZULL20090309, approved on March 9, 2009).

Sevoflurane and 2,3,5-triphenyl tetrazolium chloride (TTC) were purchased from Abbott Laboratories S.A. (Shanghai, China). Sodium pentobarbital, p-NHE1 and total NHE1 were purchased from Sigma (St. Louis, MO, USA). Antibodies against Bcl-2 (Cat. no. 2876), cleaved caspase-3 (Cat. no. 9665), Beclin-1 (Cat. no. 3495) were purchased from Cell Signaling Technology, Inc. (Danvers, MA, USA), microtubule-associated protein light chain 3 (LC3-I/II; Cat. no. ab62721-100) was purchased from Abcam, Inc. (Cambridge, England), glyceraldehyde-3-phosphate dehydrogenase (GAPDH; Cat. no. AG019) and the bicinchoninic acid (BCA) protein assay kit were purchased from Beyotime Institute of Biotechnology (Nanjing, China). NO and NOS detection assay kits were purchased from Nanjing Jiancheng Bioengineering Research Institute, Nanjing, China. NG-nitro-L-arginine methyl ester (L-NAME) was purchased from Beyotime Biotechnology Corp. (Shanghai, China). TRIzol and AMV First Strand cDNA Synthesis kits were obtained from Sangon Biotech Co., Ltd. (Shanghai, China).
Langendorff heart preparation. The hearts were prepared according to the Langendorff heart model, as described in a previous study (21). Briefly, the animals were anesthetized with pentobarbital ( $50 \mathrm{mg} / \mathrm{kg}$, intraperitoneal injection) followed by heparinization (heparin, 1,000 U/kg). The animals were then placed in a supine position, the chest cavity was opened and the hearts were removed and immediately placed in ice-cold KrebsHenseleit (K-H) buffer ( $\mathrm{pH} 7.4 ; \mathrm{KH}_{2} \mathrm{PO}_{4} 1.2 \mathrm{mM}, \mathrm{NaHCO}_{3}$ $25 \mathrm{mM}, \mathrm{KCl} 4.8 \mathrm{mM}, \mathrm{MgSO}_{4} 1.2 \mathrm{mM}$, glucose $11 \mathrm{mM}, \mathrm{NaCl}$ $118 \mathrm{mM}$ and $\mathrm{CaCl}_{2} 1.2 \mathrm{mM}$ ). The hearts were then perfused on the Langendorff apparatus at $37^{\circ} \mathrm{C}$ under a constant pressure of $80 \mathrm{mmHg}$, and continuously gassed with $95 \% \mathrm{O}_{2}$ and $5 \% \mathrm{CO}_{2}$. A water-filled balloon was inserted into the left ventricle via the left atrium, and the balloon catheter was then linked to a pressure transducer that was connected to a physiological signal acquisition system (U/4C501H Med Lab; Nanjing Meiyi Science and Technology Co., Ltd., Nanjing, China) to monitor the initial left ventricular end-diastolic pressure (LVEDP). Left ventricular developed presure (LVDP) was calculated as the difference between left ventricular systolic pressure (LVSP) and LVEDP. The work index [rate pressure product (RPP)] was calculated as the product of LVDP and heart rate (HR). All data acquisition was performed and analyzed using Chart 5 software (ADInstruments, Bella Vista, Australia).

Experimental protocol. Following $30 \mathrm{~min}$ of equilibration, the isolated rat hearts were randomly divided into the following 6 groups: i) the sham-operated control group, continuously perfused with K-H buffer for $180 \mathrm{~min}$; ii) the sevoflurane group, exposed to $2.5 \%$ sevoflurane for $15 \mathrm{~min}$, as previously descrbied (22); iii) the I/R group, exposed to global ischemia for $30 \mathrm{~min}$ followed by $120 \mathrm{~min}$ of reperfusion; iv) the sevoflurane post-conditioning (SEVOP) group, exposed to $\mathrm{I} / \mathrm{R}$ and $2.5 \%$ sevoflurane for $15 \mathrm{~min}$; v) the sevoflurane postconditioning + L-NAME group (23), exposed to I/R and $2.5 \%$ sevoflurane for $15 \mathrm{~min}$, and $100 \mu \mathrm{mol} / 1 \mathrm{~L}-\mathrm{NAME}$ for $60 \mathrm{~min}$; vi) the L-NAME group, exposed to I/R and $100 \mu \mathrm{mol} / \mathrm{l}$ L-NAME for $60 \mathrm{~min}$. The experimental protocol is illustrated in Fig. 1.

Measurement of infarct size. Myocardial infarct size was determined by TTC staining. Briefly, to visualize the unstained infarct area, the isolated hearts were removed from the Langendorff device following $2 \mathrm{~h}$ of reperfusion, cut into $5 \mu \mathrm{m}$ slices and incubated for $20 \mathrm{~min}$ in $0.1 \mathrm{~mol} / 1$ sodium phosphate buffer containing $1 \% \mathrm{TTC}$ at $37^{\circ} \mathrm{C}$. The infarct and risk zone areas were calculated with digital planimetry using Image-Pro Plus software (Media Cybernetics Inc., Rockville, MD, USA), according to the methods described in previous studies $(24,25)$.

Determination of NOS and NO levels. Following reperfusion, the hearts were removed from the Langendorff device and homogenized in ice-cold $0.9 \%$ saline solution, and then centrifuged at $600 \mathrm{x} \mathrm{g}$ for $10 \mathrm{~min}$. The NO and NOS levels were measured using a diagnostic assay kit (Nanjing Jiancheng Bioengineering Research Institute). The absorbance was determined using a DU-640 spectrophotometer (Beckman Coulter Inc., Brea, CA, USA) at $530 \mathrm{~nm}$ and plotted as a percentage of the control according to the manufacturer's instructions. 


\begin{tabular}{|c|c|c|c|c|c|}
\hline Time & $30 \mathrm{~min}$ & $30 \mathrm{~min}$ & $15 \mathrm{~min}$ & $45 \mathrm{~min}$ & $60 \mathrm{~min}$ \\
\hline SHAM & \multicolumn{5}{|c|}{ Perfusion $180 \mathrm{~min}$} \\
\hline SEVO & \multicolumn{2}{|c|}{ Perfusion } & SEVO & \multicolumn{2}{|c|}{ Perfusion } \\
\hline
\end{tabular}

\begin{tabular}{|c|c|c|c|}
\hline $\mathbf{I} / \mathbf{R}$ & Baseline & ischemia & Reperfusion $120 \mathrm{~min}$ \\
\hline SEVOP & Baseline & ischemia & Reperfusion $120 \mathrm{~min}$ \\
\hline $\begin{array}{l}\text { SEVOP+ } \\
\text { L-NAME }\end{array}$ & Baseline & ischemia & Reperfusion $120 \mathrm{~min}$ \\
\hline & & & - L-NAME \\
\hline L-NAME & Baseline & ischemia & Reperfusion $120 \mathrm{~min}$ \\
\hline
\end{tabular}

Figure 1. Experimental protocol. After randomly dividing the rats into 6 groups $(\mathrm{n}=24)$, rats underwent a 30 -min ischemia procedure, followed by 120 min of reperfusion. The sham-operated group underwent a procedure without ischemia, but it was time-matched. Sevoflurane (2.5\%) was inhaled for 15 min immediately following ischemia. Sham, sham-operated group; SEVO, sevoflurane group (no I/R injury); I/R, ischemia/reperfusion injury group; SEVOP, sevoflurane post-conditioning group; L-NAME, group treated with NG-nitro-L-arginine methyl ester (NOS inhibitor).

Detection of mitochondrial nicotinamide adenine dinucleotide $\left(N A D^{+}\right)$. The quantity of mitochondrial $\mathrm{NAD}^{+}$in the heart tissue was determined as previously described (26). Briefly, heart mitochondrial proteins were treated with ice-cold perchloric acid $(21 \%, \mathrm{v} / \mathrm{v})$ for $30 \mathrm{~min}$, centrifuged at $8,000 \mathrm{x} \mathrm{g}$, and the supernatant was then neutralized with $\mathrm{KOH}$. The $\mathrm{NAD}^{+}$content was determined by fluorometrically measuring $\mathrm{NAD}^{+}$-dependent lactate dehydrogenase activity in a reaction buffer containing $500 \mathrm{mM}$ glycine and $400 \mathrm{mM}$ hydrazine at pH 9.0 and $25^{\circ} \mathrm{C}$. Activity was evaluated at a wavelength of 340 nm (DU-640; Beckman Coulter Inc.).

Transmission electron microscopy (TEM). After teh other experiments, the isolated heart tissues were fixed with $2.5 \%$ glutaraldehyde solution for $2 \mathrm{~h}$, washed with $0.1 \mathrm{M}$ cacodylate buffer and post-fixed with $1 \%$ osmium tetroxide. Following dehydration with 50-100\% (v/v) ethanol and acetone, the sections were embedded in Epon 812 epoxy resin. Ultrathin sections were obtained at 50-70 $\mathrm{nm}$ and stained with $1 \%$ uranyl acetate and lead citrate. Apoptotic and autophagic cells were examined under a transmission electron microscope (HT7700; Hitachi, Ltd., Tokyo, Japan).

Western blot analysis. Western blot analysis was performed as previously described (27). Briefly, heart tissue (40 mg) was homogenized in lysis buffer $(0.13 \mathrm{M} \mathrm{KCl} ; 20 \mathrm{mM}$ HEPES, pH 7.4; $1 \mathrm{mM}$ EGTA; $1 \mu \mathrm{g} / \mathrm{ml}$ aprotinin; $1 \mu \mathrm{g} / \mathrm{ml}$ leupeptin; and $1 \mathrm{mM}$ PMSF). Protein concentrations were quantified using a BCA protein assay kit. Protein samples $(50 \mu \mathrm{g})$ were resolved on a sodium dodecyl sulfate polyacrylamide gel and then transferred onto nitrocellulose membranes. The membranes were blocked for $2 \mathrm{~h}$ at room temperature with $5 \%$ milk in Tris-buffered saline, followed by incubation with the following primary antibodies at $4^{\circ} \mathrm{C}$ overnight: $\mathrm{Bcl}-2$ (1:1,000 dilution), cleaved caspase-3 (1:1,000 dilution), Beclin-1 (1:1,000 dilution), LC3-I (1:1,000 dilution), LC3-II (1:1,000 dilution), NHE1 (1:1,000 dilution), p-NHE1 (1:1,000 dilution) and GAPDH (1:1,000 dilution). After washing 3 times with $0.05 \%$ TBS and Tween-20, the membranes were incubated with goat anti-rabbit secondary antibodies conjugated with horseradish peroxidase (A0216; Beyotime Institute of Biotechnology), at a dilution of 1:2,000. Bands were detected using the Pierce ECL detection system (Pierce Biotechnology, Thermo Fisher Scientific Inc., Waltham, MA, USA). Band intensity was quantified using ImageJ software (National Institutes of Health, Bethesda, MD, USA). Protein expression was quantified relative to GAPDH bands from the same sample.

Reverse transcription-quantitative polymerase chain reaction $(R T-q P C R)$. RT-qPCR was used to measure the NHE1 mRNA expression levels. Briefly, the hearts were harvested following reperfusion, total RNA was extracted from the left ventricular heart tissues using TRIzol reagent and the RNA purity was quantified spectrophotometrically at a ratio of 260 to $280 \mathrm{~nm}$. cDNA was obtained from $1 \mu \mathrm{g}$ total RNA using the AMV First Strand cDNA Synthesis kit according to the manufacturer's instructions (New England Biolabs Inc., Ipswich, MA, USA). Quantitative (real-time) PCR (qPCR) was performed using iQ SYBR-Green Supermix (Bio-Rad Laboratories, Inc., Hercules, CA, USA). The NHE1 and GAPDH (internal control) primer sequences were defined as follows: NHE1 forward, 5'-GCGGCGAGCAGATCAATAA-3' and reverse, 5'-ACAGT GACGGCATCGTTGAG-3'; and GAPDH forward, 5'-CAAGT TCAACGGCACAGTCAA-3' and reverse, 5'-CGCCAGTAGA CTCCACGACA-3'. The reaction conditions were as follows: $10 \mathrm{sec}$ at $95^{\circ} \mathrm{C}, 40 \mathrm{sec}$ at $60^{\circ} \mathrm{C}$ and $45 \mathrm{sec}$ at $72^{\circ} \mathrm{C}$ for 40 cycles. Amplification of the products was followed by melting curve analysis using Applied Biosystems 7500 system software, as previously described (28) (Life Technologies, Grand Island, NY, USA). The value of NHE1 mRNA was expressed relative to that of GAPDH from the same sample. 

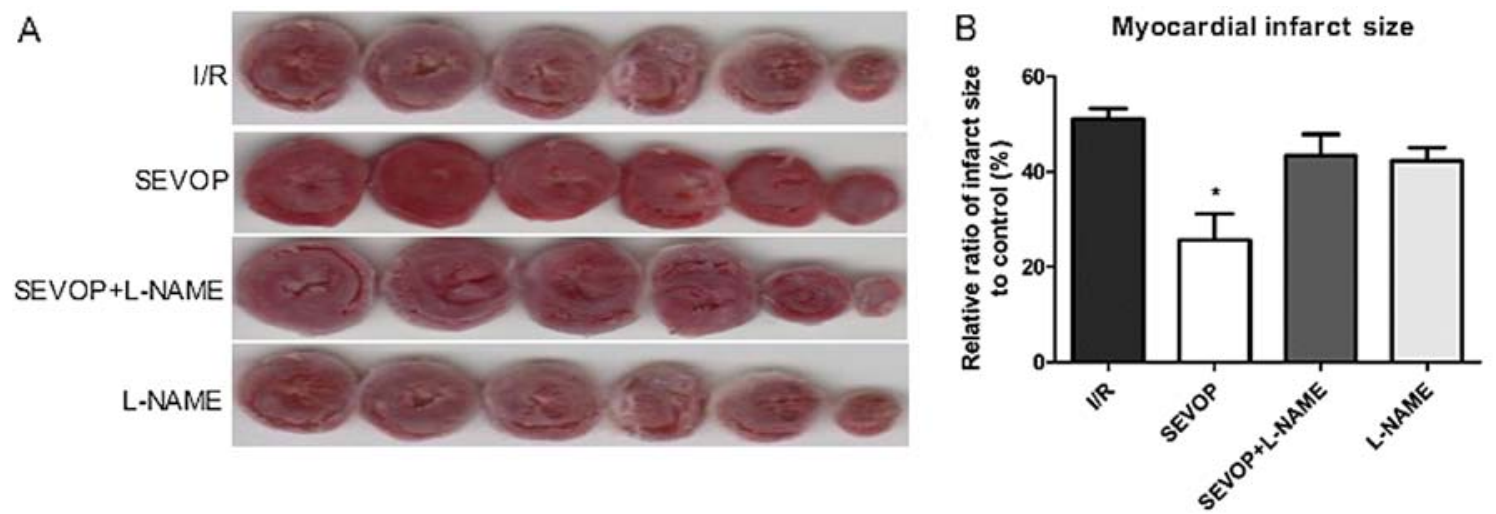

Figure 2. Sevoflurane post-conditioning decreases infarct size which is increased by myocardial ischemia/reperfusion injury (MIRI) in rats. (A) Representative TTC-stained images and (B) quantification of the percentage of the injured area. The columns and error bars represent the means \pm SD; $n=6$ experiments. A oneway ANOVA followed by Tukey's post-hoc test was used to determine statistical significance. " $\mathrm{P}<0.05 \mathrm{vs}$. I/R. Sham, sham-operated group; SEVO, sevoflurane group (no I/R injury); I/R, ischemia/reperfusion injury group; SEVOP, sevoflurane post-conditioning group; L-NAME, group treated with NG-nitro-L-arginine methyl ester (NOS inhibitor); TTC, triphenyltetrazolium chloride.
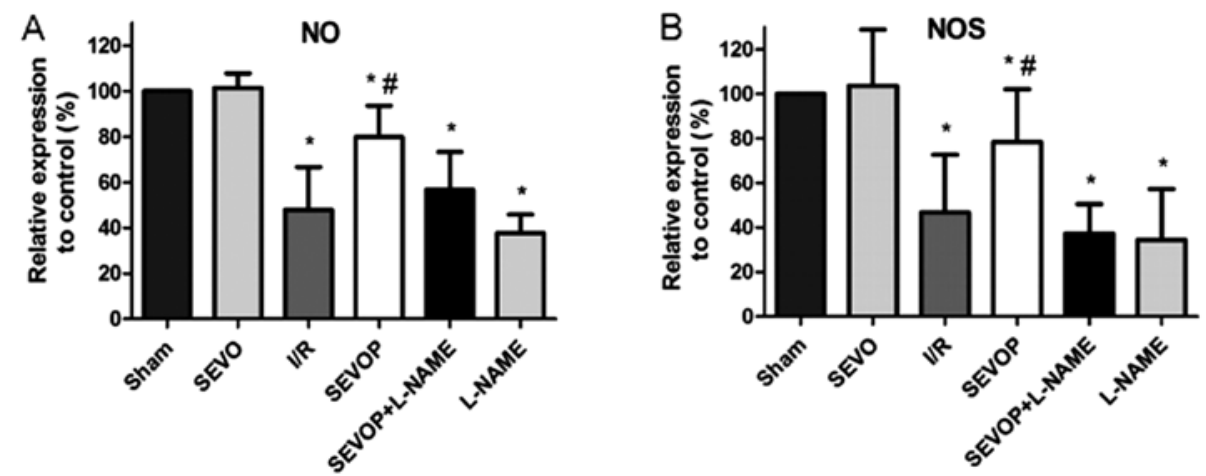

Figure 3. Sevoflurane post-conditioning increases nitric oxide (NO) and nitric oxide synthase (NOS) production following ischemia/reperfusion. (A) Sevoflurane post-conditioning increased NO production. (B) Sevoflurane post-conditioning increased NOS production. Sevoflurane post-conditioning-induced NO and NOS production was inhibited by L-NAME treatment. Data are presented as the means \pm SD; $n=6$ experiments. A one-way ANOVA followed by Tukey's post-hoc test was used to determine statistical significance. ${ }^{*} \mathrm{P}<0.05$ vs. sham. ${ }^{*} \mathrm{P}<0.05$ vs. I/R. Sham, sham-operated group; SEVO, sevoflurane group (no I/R injury); I/R, ischemia/reperfusion injury group; SEVOP, sevoflurane post-conditioning group; L-NAME, group treated with NG-nitro-L-arginine methyl ester (NOS inhibitor).

Statistical analysis. All data are expressed as the means \pm standard deviation (SD) and analyzed by one-way ANOVA followed by Tukey's post-hoc test for multiple comparisons. A value of $\mathrm{P}<0.05$ (two-tailed) was considered to indicate a statistically significant difference. All statistical analyses were conducted using GraphPad Prism 5.0 software (GraphPad Software Inc., San Diego, CA, USA).

\section{Results}

Sevoflurane post-conditioning reduces the myocardial infarct size. To evaluate the effects of sevoflurane post-conditioning on MIRI in vitro, we measured rat myocardial infarct size using the TTC staining method, as previously described (25). Following $120 \mathrm{~min}$ of reperfusion, the infarct size in the sevoflurane post-conditioning treatment group (SEVOP) decreased to $25.67 \pm 5.52 \%$ compared to that in the $\mathrm{I} / \mathrm{R}$ group (I/R, no treatment, 51.07 $\pm 2.27 \%$; P<0.05). Treatment with L-NAME abolished the reducing effects of sevoflurane post-conditioning on the infarct size. Notably, without sevoflurane postconditioning, treatment with L-NAME alone did not affect the ratio of the infarct size when compared with the untreated groups ( $\mathrm{P}>0.05$; Fig. 2 ).
Sevoflurane post-conditioning upregulates the NOS and NO levels. We measured the NO and NOS levels in the heart tissues subjected to MIRI with or without sevoflurane post-conditioning. We observed that the NO and NOS levels were decreased in the heart tissues subjected to MIRI when compared with the levels in the sham-operated group $(\mathrm{P}<0.05, \mathrm{n}=6$ experiments; Fig. 3$)$. However, sevoflurane post-conditioning induced a significant increase in the NO and NOS levels when compared with the $\mathrm{I} / \mathrm{R}$ group (no treatment, $\mathrm{P}<0.05, \mathrm{n}=6$ experiments; Fig. 3 ). The increase in the levels of NO and NOS induced by sevoflurane post-conditioning was markedly suppressed by treatment with L-NAME (Fig. 3).

Sevoflurane post-conditioning inhibits the $I / R$ induced loss in $N A D^{+}$content. Mitochondrial $\mathrm{NAD}^{+}$levels may be used as a marker of MPTP opening in MIRI heart tissues and it has been suggested that $\mathrm{NAD}^{+}$levels are significantly decreased in tissues subjected to MIRI (29). In this study, to confirm MPTP opening in injured heart tissue during reperfusion, as well as the protective effects of sevoflurane post-conditioning on this process, we measured the $\mathrm{NAD}^{+}$levels in the mitochondria isolated from heart tissues. The $\mathrm{NAD}^{+}$levels were significantly decreased following exposure to $\mathrm{I} / \mathrm{R}$ compared to the 
Table I. Determination of NAD ${ }^{+}$content.

\begin{tabular}{|c|c|c|c|c|c|c|}
\hline Groups & Sham & SEVO & $\mathrm{I} / \mathrm{R}$ & SEVOP & SEVOP + L-NAME & L-NAME \\
\hline $\mathrm{NAD}^{+}(\mathrm{nmol} / \mathrm{g})$ & $178 \pm 37$ & $181 \pm 40$ & $91 \pm 27^{\mathrm{a}}$ & $122 \pm 30^{\mathrm{a}, \mathrm{b}}$ & $95 \pm 26^{\mathrm{a}}$ & $88 \pm 24^{\mathrm{a}}$ \\
\hline
\end{tabular}

${ }^{\mathrm{a} P}<0.05$ vs. SEVOP group; ${ }^{\mathrm{b}} \mathrm{P}<0.05$ vs. I/R group. Sham, sham-operated group; SEVO, sevoflurane group (no I/R injury); I/R, ischemia/reperfusion injury group; SEVOP, sevoflurane post-conditioning group; L-NAME, group treated with NG-nitro-L-arginine methyl ester.

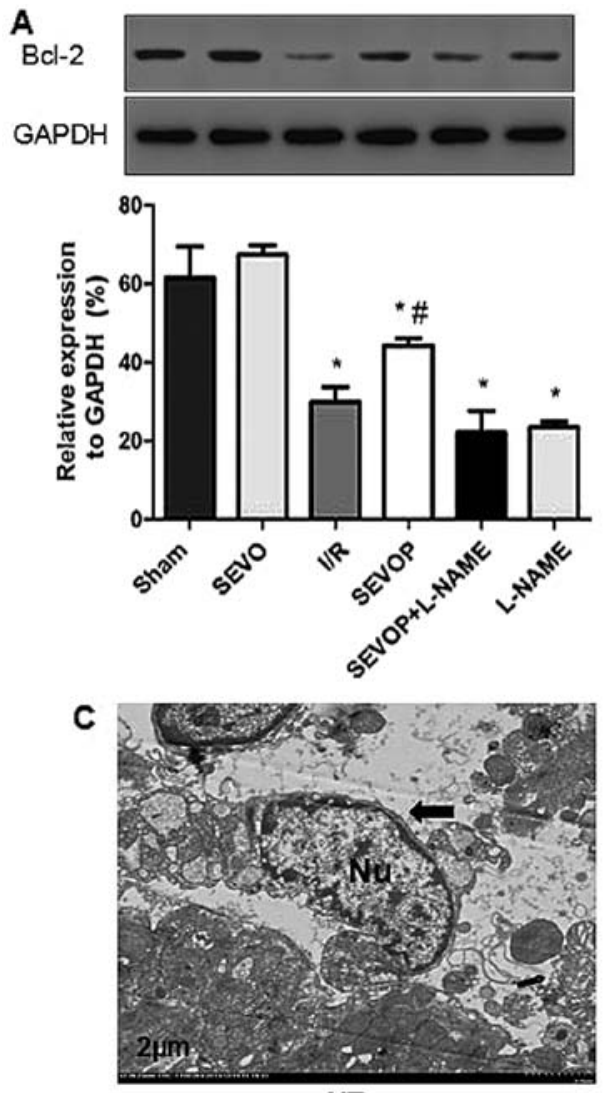

I/R
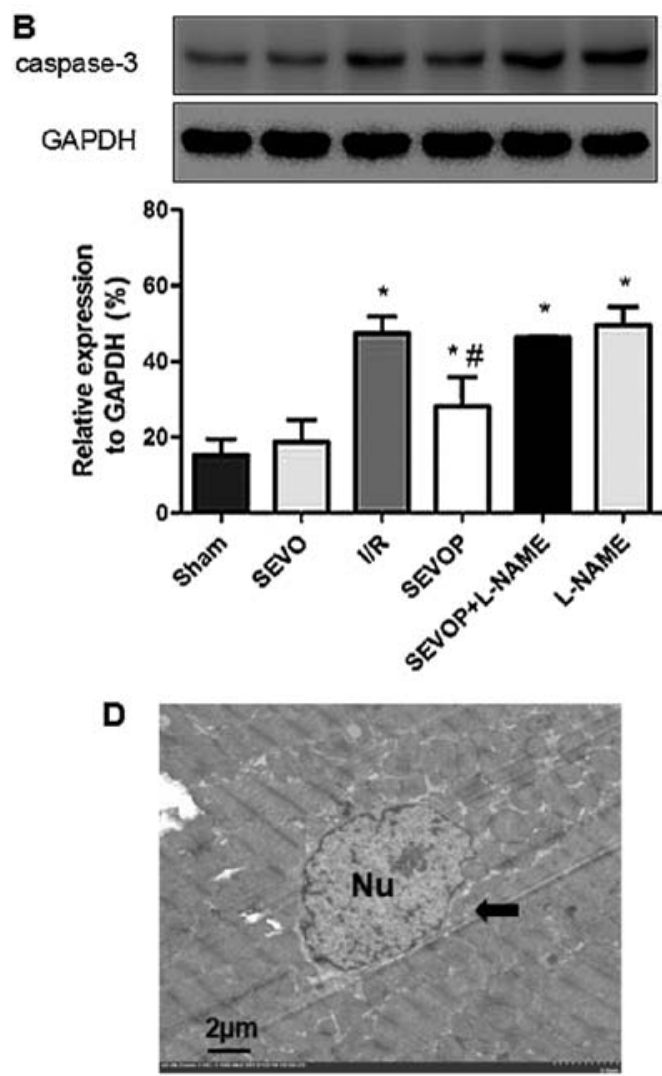

SEVOP

Figure 4. Alterations in Bcl-2 expression and cleaved-caspase-3 activity following sevoflurane post-conditioning. Shown are myocardial western blots of Bcl-2 and cleaved caspase-3 expression. (A and B) Representative western blots of Bcl-2 and cleaved-caspase-3, respectively, in Sham, SEVO, I/R, SEVOP, SEVOP+L-NAME and L-NAME groups. (C and D) Representative transmission electron microscopy images from the $\mathrm{I} / \mathrm{R}$ and SEVOP groups, respectively (original magnification, x2000; scale bar, $2 \mu \mathrm{m}$ ). Images show that apoptosomes were decreased in the SEVOP group when compared with the I/R group. As indicated by the arrows, panel (C) shows mild swelling of the nucleus, chromatin edge set along the nuclear membrane, loose cytoplasm and moderate swelling of the mitochondria These effects were attenuated in the SEVOP group (D). The data are presented as the means \pm SD ( $n=5$ experiments). A one-way ANOVA followed by Tukey's post-hoc test was used to determine statistical significance. ${ }^{~} \mathrm{P}<0.05$ vs. sham. ${ }^{~} \mathrm{P}<0.05$ vs. I/R. SEVO, sevoflurane group (no I/R injury); I/R, ischemia/reperfusion injury group; SEVOP, sevoflurane post-conditioning group; L-NAME, group treated with NG-nitro-L-arginine methyl ester (NOS inhibitor); Nu, nucleus.

group exposed to sevoflurane post-conditioning (181 \pm 40 to $91 \pm 27 \mathrm{nmol} / \mathrm{g})$. The I/R-induced loss in the $\mathrm{NAD}^{+}$content in the heart tissues subjected to MIRI was reversed by sevoflurane post-conditioning (from $91 \pm 27$ to $122 \pm 30 \mathrm{nmol} / \mathrm{g}$ ), and this effect was largely blocked by treatment with L-NAME (Table I).

Sevoflurane post-conditioning decreases myocardial tissue apoptosis following MIRI. Western blot analysis and quantitative analysis indicated that the Bcl-2 levels were significantly lower in the I/R group $(29.92 \pm 3.75)$ when compared with the sham-operated group (61.54 $\pm 7.89 ; \mathrm{P}<0.05 ;$ Fig. 4A). Sevoflurane post-conditioning increased Bcl-2 expression (44.32 \pm 1.75$)$; however, this effect was inhibited by treatment with
L-NAME (22.17 \pm 5.47$)$, indicating that sevoflurane post-conditioning inhibited I/R-induced apoptosis. The results obtained for the other apoptotic marker, cleaved caspase-3 (Fig. 4B), also indicated that sevoflurane post-conditioning inhibited apoptosis. I/R injury increased the levels of cleaved caspase-3, and sevoflurane post-conditioning decreased the cleaved caspase-3 levels. Once again, these effects were reversed by treatment with L-NAME (Fig. 4B). We also used TEM to observe apoptosome formation and confirmed reduced apoptosome formation following sevoflurane post-conditioning. The ultrastructure of the myocardial tissues exhibited a variety of characteristics associated with apoptosis, including mild swelling of the nucleus, chromatin edge set along the nuclear membrane, a 

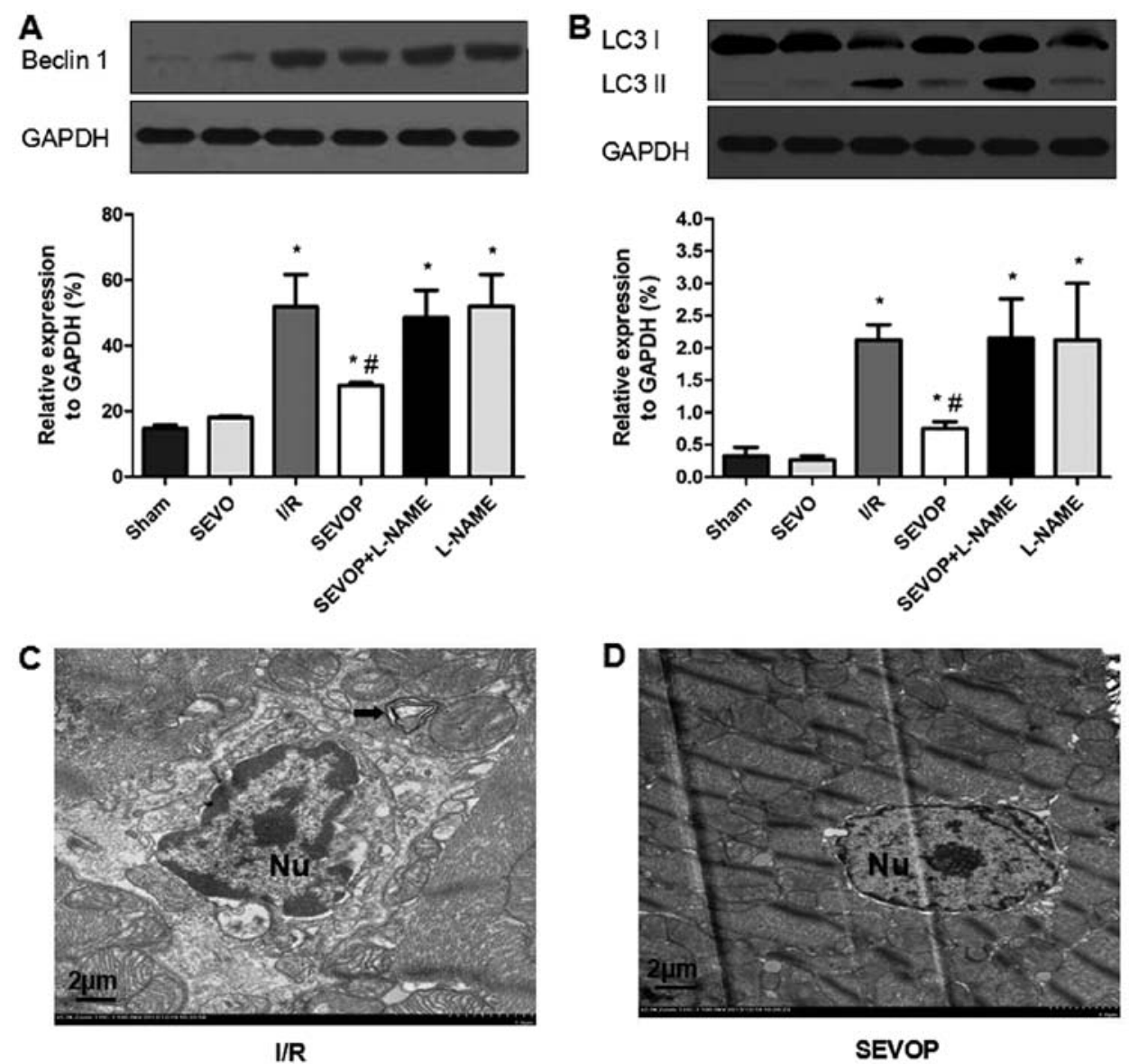

Figure 5. Alterations in LC3-II/I and Beclin-1 expression following sevoflurane post-conditioning. Shown are myocardial western blots of LC3-II/I and Beclin-1 expression. (A and B) Representative western blots of Beclin-1 and LC3II/I, respectively, in the Sham, SEVO, I/R, SEVOP, SEVOP + L-NAME and L-NAME groups. Values are expressed as the means $\pm \mathrm{SD}$ ( $\mathrm{n}=5$ experiments). A one-way ANOVA followed by Tukey's post-hoc test was used to determine statistical significance. "P $<0.05$ vs Sham. ${ }^{~} \mathrm{P}<0.05$ vs I/R. (C and D) Representative transmission electron microscopy images from the I/R and SEVOP groups, respectively (original magnification, $\mathrm{x} 2,000$; scale bar, $2 \mu \mathrm{m}$ ), showing that the number of autophagic vacuoles (AVs) decreased in the sevoflurane post-conditioning group when compared with the I/R group. (C) Arrow indicates autophagic vacuole. Sham, sham-operated group; SEVO, sevoflurane group (no I/R injury); I/R, ischemia/reperfusion injury group; SEVOP, sevoflurane post-conditioning group; L-NAME, group treated with NG-nitro-L-arginine methyl ester (NOS inhibitor); Nu, nucleus.

loose cytoplasm, moderate mitochondrial swelling and sparse cristae (Fig. 4C). However, sevoflurane post-conditioning reversed these effects (Fig. 4D). These results confirm the hypothesis that MIRI increases apoptosis in the heart and that sevoflurane post-conditioning reduces MIRI-induced apoptosis in myocardial tissue.

Sevoflurane post-conditioning inhibits autophagy following MIRI. To examine whether the protective effects if sevoflurane post-conditioning in MIRI are mediated by the inhibition of autophagy in vivo, we detected the expression of the autophagic markers, LC3-I/II and Beclin-1. Western blot and quantitative analyses indicated that the LC3-I/II and Beclin-1 levels were significantly higher in the $\mathrm{I} / \mathrm{R}$ group when compared with the sham-operated group (Fig. 5A and B). However, sevoflurane post-conditioning decreased Beclin-1 and LC3-I/II expression (Fig. 5A and B). However, treatment with L-NAME largely abolished these effects, suggesting that sevoflurane post-conditioning reduces the I/R-mediated activation of autophagy (Fig. 5A and B). We also used TEM to detect the formation of autophagosomes and double limiting membranes in the I/R group. Ultrastructural image analysis revealed that the number of autophagic vacuoles (AVs) was markedly increased in the heart tissue subjected to I/R (Fig. 5C). Sevoflurane postconditioning decreased the number of AVs (Fig. 5D).

Protein expression of $p$-NHE1 and NHE1 mRNA expression is inhibited in myocardial tissues subjected to I/R following sevoflurane post-conditioning. We investigated whether the NHE1 phosphorylation levels are altered in myocardial tissues subjected to I/R. We measured the protein expression levels of p-NHE1 and total (t-)NHE1 by western blot analysis. The ratio of p-NHE1 to NHE1 protein increased significantly in the I/R group when compared with the sham-operated group. p-NHE1 protein expression was significantly decreased following sevoflurane post-conditioning. However, treatment with L-NAME abolished the reducing effects of sevoflurane post-conditioning on p-NHE1 expression (Fig. 6). Furthermore, the results of RT-qPCR confirmed the significant increase in NHE1 mRNA expression in the I/R group compared with the sham-operated group $(\mathrm{P}<0.05)$. Moreover, when compared with the untreated I/R group, the NHE1 mRNA levels were decreased in the sevoflurane post-conditioning group and were restored in the L-NAME treatment group (Fig. 7). 
A
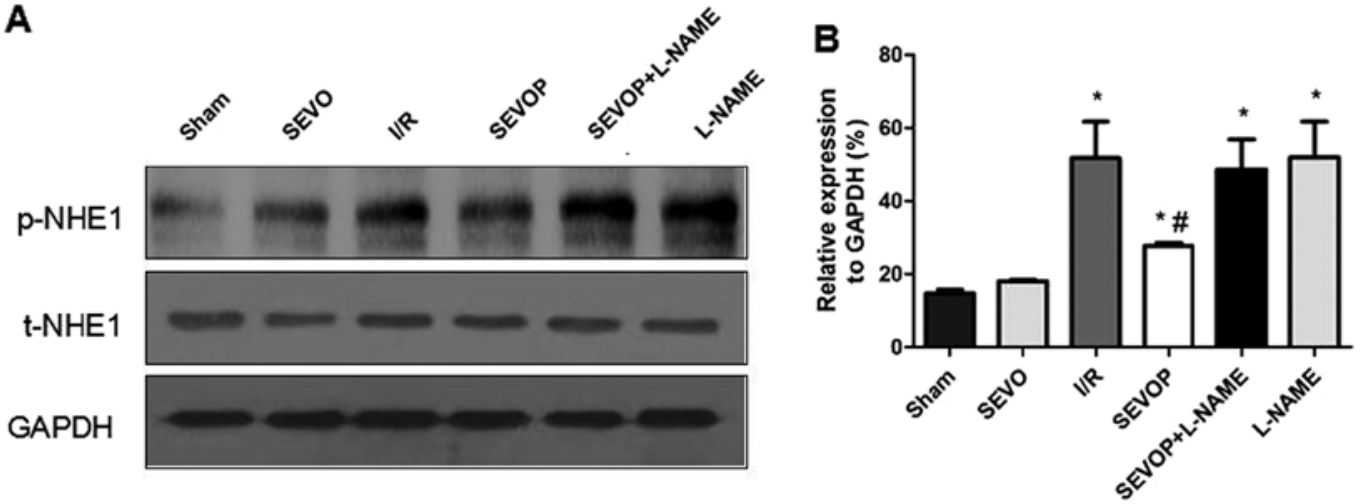

Figure 6. Sevoflurane post-conditioning inhibits p-NHE1 expression. (A and B) Shown are myocardial western blots of phosphorylated (p-)and total (t-)NHE1 expression. Values are expressed as the means \pm SD. A one-way ANOVA followed by Tukey's post-hoc test was used to determine statistical significance. ${ }^{P}<0.05$ vs. Sham. "P<0.05 vs. I/R. Sham, sham-operated group; SEVO, sevoflurane group (no I/R injury); I/R, ischemia/reperfusion injury group; SEVOP, sevoflurane post-conditioning group; L-NAME, group treated with NG-nitro-L-arginine methyl ester (NOS inhibitor).
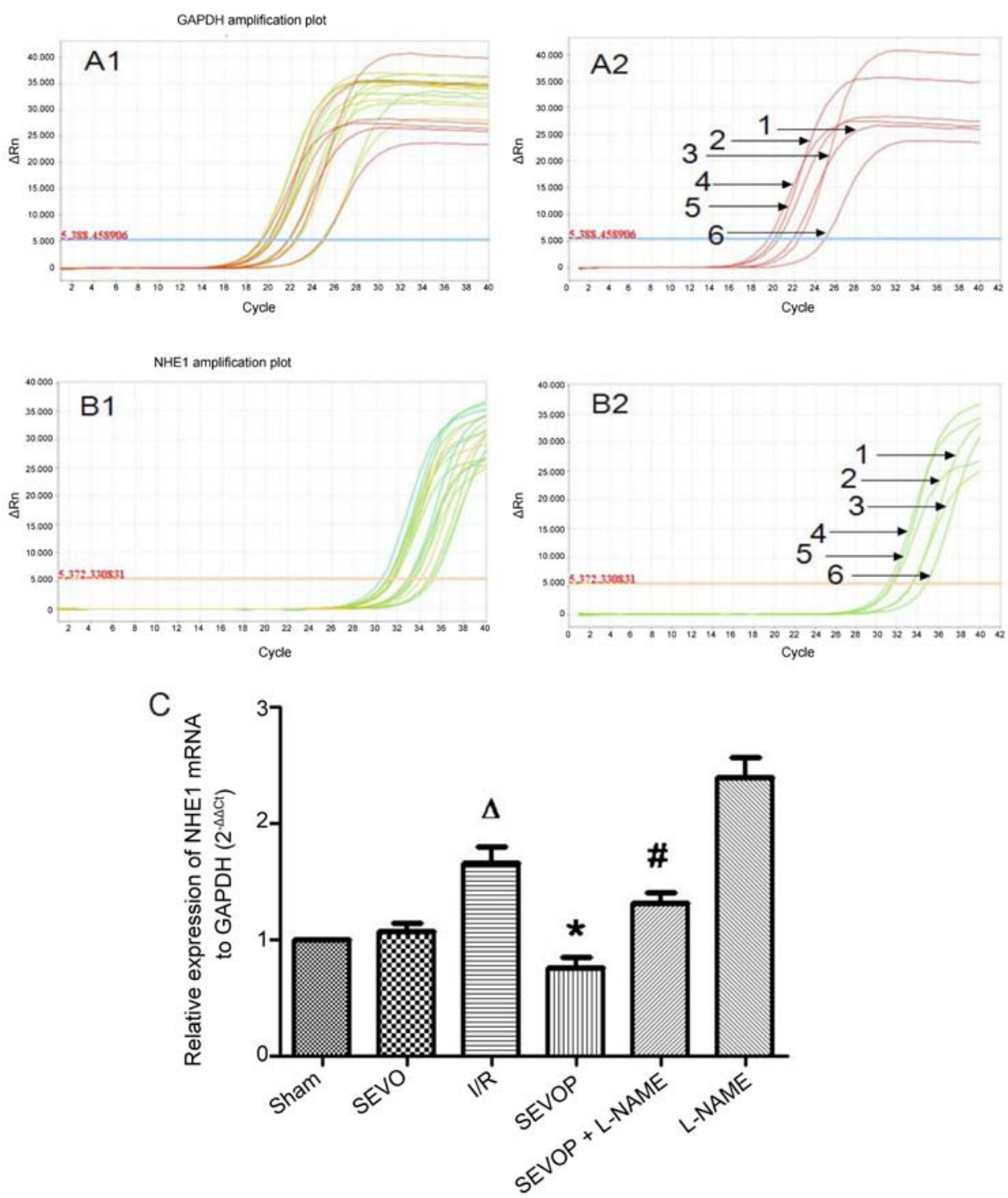

Figure 7. Sevoflurane post-conditioning inhibits NHE1 mRNA expression. (A1) Representative amplification plot of GAPDH; 3 microwells/group, 6 groups; 3 different colors indicate 3 microwells. (A2) Representative amplification plot of GAPDH for 6 groups. Numbers 1, 2, 3, 4, 5 and 6, represent the amplification plot of GAPDH for the Sham, SEVO, I/R, SEVOP, SEVOP + L-NAME and L-NAME groups, respectively. (B1) Representative amplification plot of NHE1; 3 microwells/ group, 6 groups; 3 different colors indicate 3 microwells. (B2) Representative amplification plot of NHE1 for 6 groups. Numbers 1,2,3,4,5 and 6, represent the amplification plot of NHE1 for the Sham, SEVO, I/R, SEVOP, SEVOP + L-NAME and L-NAME groups, respectively. (C) mRNA expression of NHE1 relative to GAPDH, in the Sham, SEVO, I/R, SEVOP, SEVOP + L-NAME and L-NAME groups, respectively. Values are expressed as the means \pm SD ( $=3$ ). A one-way ANOVA followed by Tukey's post-hoc test was used to determine statistical significance. ${ }^{\Delta} \mathrm{P}<0.05$ vs. Sham, ${ }^{*} \mathrm{P}<0.05$ vs. I/R, ${ }^{\prime \prime} \mathrm{P}<0.05$ vs. SEVOP. . 


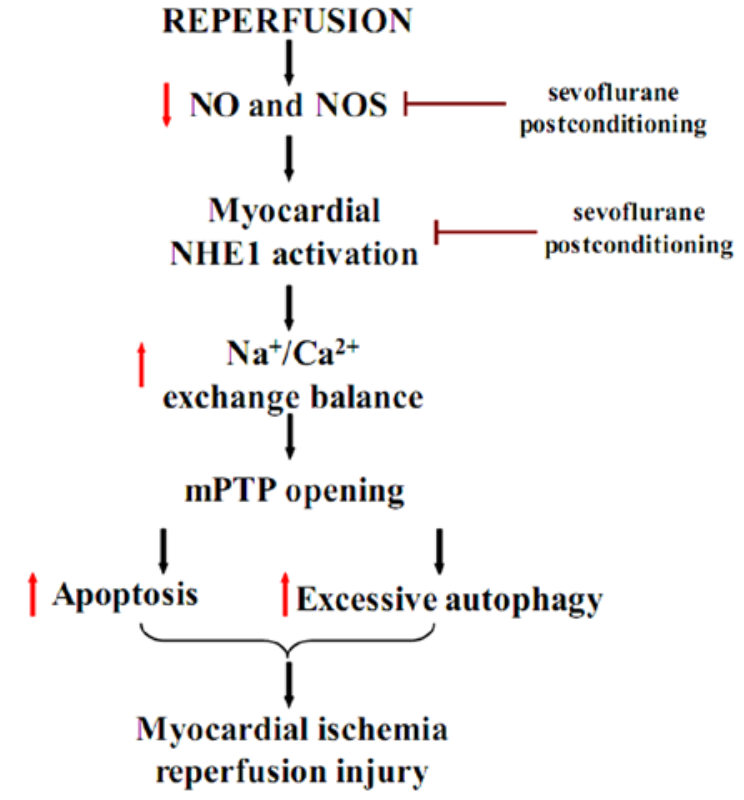

Figure 8. Proposed mechanism of sevoflurane post-conditioning cardioprotection against myocardial ischemia/reperfusion injury (MIRI). Black arrows indicate transition; red downward arrows indicate downregulation; red upward arrows indicate upregulation; and red bar-headed lines indicate inhibition.

\section{Discussion}

In the present study, we used a rat model of MIRI nvestigate a novel mechanism through which sevoflurane post-conditioning protects the heart against MIRI. Our results confirmed I/R induced apoptosis and excessive autophagy, eventually causing myocardial infarction. Notably, sevoflurane post-conditioning significantly attenuated MIRI and inhibited apoptosis and excessive autophagy. Furthermore, the NHE1 phosphorylation and mRNA expression levels were markedly downregulated by sevoflurane post-conditioning. These effects were all abolished by treatment with L-NAME.

To the best of our knowledge, the present study is the first to demonstrate that sevoflurane post-conditioning reduces MIRI in vitro, and furthermore, that this protective mechanism is mediated through an increase in NOS and a decrease in p-NHE1 levels.

Apoptosis is one of the major mechanisms responsible for cell death in MIRI $(28,30)$. The inhibition of apoptosis has been demonstrated to be a key mechanisms that reduces myocardial infarct size $(31,32)$. Additionally, it has been suggested that the appropriate upregulation of autophagy exerts cardioprotective effects during I/R injury (33). However, other studies have demonstrated that certain drugs, such as $\alpha$-lipoic acid, protect the heart against injury by inhibiting excessive autophagy (34). In the present study, we observed that sevoflurane post-conditioning elevated Bcl-2 expression, decreased the Beclin-1 and LC3-I/II levels, and attenuated I/R injury. As confirmed by by our TEM observations, our results suggest that sevoflurane post-conditioning protects the heart tissue following myocardial infarction and reduces MIRI through the inhibition of apoptosis and excessive autophagy.

$\mathrm{NO}$, which is generated from L-arginine by NOS1, NOS2 and NOS3, is a key factor which has been demonstrated to exert protective effects against cardiac injury $(35,36)$. Previous studies have also demonstrated that $\mathrm{NO}$ is associated with the pathogenesis of MIRI (37) and that it regulates cytoprotection during I/R injury (38). In the present study, both NO and NOS levels expression were significantly increased by sevoflurane post-conditioning when compared with the I/R group. We also found that treatment with L-NAME attenuated the effects of sevoflurane post-conditioning on the NO and NOS levels. This indicates that NOS regulates the $\mathrm{NO} / \mathrm{ONOO}^{-}$balance and mediates the protective effects of sevoflurane post-conditioning in MIRI.

The inhibition of MPTP opening is a potential cardioprotective mechanism. Thus, to investigate the role of MPTP opening in MIRI following sevoflurane post-conditioning, we measured the $\mathrm{NAD}^{+}$levels, which indirectly represent MPTP opening (14). Compared with the sham-operated group, the $\mathrm{NAD}^{+}$levels were significantly decreased in the I/R group; however, sevoflurane post-conditioning inhibited the loss of $\mathrm{NAD}^{+}$(Table I). We also observed that treatment with L-NAME inhibited the effects of sevoflurane post-conditioning on the $\mathrm{NAD}^{+}$levels.

MIRI has been demonstrated to be associated with a variety of significant intracellular and extracellular metabolic alterations, including elevated potassium, increased lactate and acidosis $(39,40)$. NHE is a membrane transport protein that is activated by ischemia and that catalyzes the exchange of $\mathrm{Na}^{+}$for $\mathrm{H}^{+}$. Intracellular $\mathrm{Na}^{+}$and $\mathrm{Ca}^{2+}$ overload may lead to cardiac injury following myocardial ischemia. Recent studies have confirmed that NHE1 inhibition prevents MPTP opening and reduces myocardial ischemia injury $(40,41)$. In the present study, sevoflurane post-conditioning reduced the phosphorylation and transcription levels of NHE1, whereas L-NAME inhibited these effects (Fig. 7). However, when compared with the I/R group, treatment with L-NAME alone did not affect the phosphorylated NHE1 protein or NHE1 mRNA levels.

In conclusion, the findings of the present study confirm the cardioprotective effects of sevoflurane post-conditioning against MIRI through the inhibition of apoptosis and excessive autophagy. The protective effects of sevoflurane post-conditioning following I/R injury in vitro may be regulated by the increase in NOS and the decrease in p-NHE1 levels (Fig. 8). The present study provides evidence for a new protective mechanism of sevoflurane post-conditioning against MIRI. The mechanisms reported in this study may aid the future design of therapeutic strategies to prevent MIRI in humans and therefore, further clinical studies are warranted to explore optimal sevoflurane post-conditioning treatment conditions.

\section{Acknowledgements}

The present study was supported by the National Natural Science Foundation of China (grant no. 81372024, to J.Z.), the Natural Science Foundation of Jiangsu Province, China (grant no. BK20141187, to C.W), and the Science and Technology Development Plan of Suzhou City, China (grant no. SYSD2012085, to J.C.)

\section{References}

1. Hu Q, Chen J, Jiang C and Liu HF: Effect of peroxisome proliferator-activated receptor gamma agonist on heart of rabbits with acute myocardial ischemia/reperfusion injury. Asian Pac J Trop Med 7: 271-275, 2014. 
2. Zhu M, Feng J, Lucchinetti E, Fischer G, Xu L, Pedrazzini T, Schaub MC and Zaugg M: Ischemic postconditioning protects remodeled myocardium via the PI3K-PKB/Akt reperfusion injury salvage kinase pathway. Cardiovasc Res 72: 152-162, 2006.

3. Xia A, Xue Z, Wang W, Zhang T, Wei T, Sha X, Ding Y and Zhou W: Naloxone postconditioning alleviates rat myocardial ischemia reperfusion injury by inhibiting JNK activity. Korean J Physiol Pharmacol 18: 67-72, 2014.

4. Yao YY, Zhu MH, Zhang FJ, Wen CY, Ma LL, Wang WN, Wang CC, Liu XB, Yu LN, Qian LB, et al: Activation of Akt and cardioprotection against reperfusion injury are maximal with only five minutes of sevoflurane postconditioning in isolated rat hearts. J Zhejiang Univ Sci B 14: 511-517, 2013.

5. Sakai EM, Connolly LA and Klauck JA: Inhalation anesthesiology and volatile liquid anesthetics: Focus on isoflurane, desflurane, and sevoflurane. Pharmacotherapy 25: 1773-1788, 2005.

6. Zheng Z, Yang M, Zhang F, Yu J, Wang J, Ma L, Zhong Y, Qian L, Chen G, Yu L and Yan M: Gender-related difference of sevoflurane postconditioning in isolated rat hearts: Focus on phosphatidylinositol-3-kinase/Akt signaling. J Surg Res 170: e3-e9, 2011.

7. Inamura Y, Miyamae M, Sugioka S, Domae N and Kotani J: Sevoflurane postconditioning prevents activation of caspase 3 and 9 through antiapoptotic signaling after myocardial ischemiareperfusion. J Anesth 24: 215-224, 2010.

8. Ceyhan D, Tanriverdi B and Bilir A: Comparison of the effects of sevoflurane and isoflurane on myocardial protection in coronary bypass surgery. Anadolu Kardiyol Derg 11: 257-262, 2011.

9. Yao YT, Li LH, Chen L, Wang WP, Li LB and Gao CQ: Sevoflurane postconditioning protects isolated rat hearts against ischemia-reperfusion injury: The role of radical oxygen species, extracellular signal-related kinases $1 / 2$ and mitochondrial permeability transition pore. Mol Biol Rep 37: 2439-2446, 2010.

10. Gong JS, Yao YT, Fang NX and Li LH: Sevoflurane postconditioning attenuates reperfusion-induced ventricular arrhythmias in isolated rat hearts exposed to ischemia/reperfusion injury. Mol Biol Rep 39: 6417-6425, 2012.

11. Yao Y, Li L, Li L, Gao C and Shi C: Sevoflurane postconditioning protects chronically-infarcted rat hearts against ischemia-reperfusion injury by activation of pro-survival kinases and inhibition of mitochondrial permeability transition pore opening upon reperfusion. Biol Pharm Bull 32: 1854-1861, 2009.

12. Zweier JL, Flaherty JT and Weisfeldt ML: Direct measurement of free radical generation following reperfusion of ischemic myocardium. Proc Natl Acad Sci USA 84: 1404-1407, 1987.

13. Yoshida A, Asanuma H, Sasaki H, Sanada S, Yamazaki S, Asano Y, Shinozaki Y, Mori H, Shimouchi A, Sano M, et al: $\mathrm{H}_{2}$ mediates cardioprotection via involvements of $\mathrm{K}_{\mathrm{ATP}}$ channels and permeability transition pores of mitochondria in dogs. Cardiovasc Drugs Ther 26: 217-226, 2012.

14. Petrosillo G, Di Venosa N, Moro N, Colantuono G, Paradies V, Tiravanti E, Federici A, Ruggiero FM and Paradies G: In vivo hyperoxic preconditioning protects against rat-heart ischemia/reperfusion injury by inhibiting mitochondrial permeability transition pore opening and cytochrome c release. Free Radic Biol Med 50: 477-483, 2011

15. Prasad V, Lorenz JN, Miller ML, Vairamani K, Nieman ML, Wang Y and Shull GE: Loss of NHE1 activity leads to reduced oxidative stress in heart and mitigates high-fat diet-induced myocardial stress. J Mol Cell Cardiol 65: 33-42, 2013.

16. Goldman A, Shahidullah M, Goldman D, Khailova L, Watts G Delamere $\mathrm{N}$ and Dvorak K: A novel mechanism of acid and bile acid-induced DNA damage involving $\mathrm{Na}^{+} / \mathrm{H}^{+}$exchanger: implication for Barrett's oesophagus. Gut 59: 1606-1616, 2010.

17. Qian GQ, Peng X, Cai C and Zhao GP: Effect on eNOS/NO Pathway in MIRI rats with preconditioning of GFPC from Dang Gui Si Ni decoction. Pharmacognosy Res 6: 133-137, 2014.

18. Nakamura TY, Iwata Y, Arai Y, Komamura K and Wakabayashi S: Activation of $\mathrm{Na}^{+} / \mathrm{H}^{+}$exchanger 1 is sufficient to generate $\mathrm{Ca}^{2+}$ signals that induce cardiac hypertrophy and heart failure. Circ Res 103: 891-899, 2008

19. Iwamoto T, Wakabayashi S and Shigekawa M: Growth factorinduced phosphorylation and activation of aortic smooth muscle $\mathrm{Na}^{+} / \mathrm{Ca}^{2+}$ exchanger. J Biol Chem 270: 8996-9001, 1995.

20. Villa-Abrille MC, Cingolani E, Cingolani HE and Alvarez BV: Silencing of cardiac mitochondrial NHE1 prevents mitochondrial permeability transition pore opening. Am J Physiol Heart Circ Physiol 300: H1237-H1251, 2011.

21. Testai L, Martelli A, Cristofaro M, Breschi MC and Calderone V: Cardioprotective effects of different flavonoids against myocardial ischaemia/reperfusion injury in Langendorff-perfused rat hearts. J Pharm Pharmacol 65: 750-756, 2013.
22. Deyhimy DI, Fleming NW, Brodkin IG and Liu H: Anesthetic preconditioning combined with postconditioning offers no additional benefit over preconditioning or postconditioning alone. Anesth Analg 105: 316-324, 2007.

23. Burley DS and Baxter GF: B-type natriuretic peptide at early reperfusion limits infarct size in the rat isolated heart. Basic Res Cardiol 102: 529-541, 2007.

24. Lecour S, Smith RM, Woodward B, Opie LH, Rochette L and Sack MN: Identification of a novel role for sphingolipid signaling in TNF alpha and ischemic preconditioning mediated cardioprotection. J Mol Cell Cardiol 34: 509-518, 2002.

25. Deng C, Sun Z, Tong G, Yi W, Ma L, Zhao B, Cheng L, Zhang J, Cao $\mathrm{F}$ and $\mathrm{Yi} \mathrm{D}: \alpha$-Lipoic acid reduces infarct size and preserves cardiac function in rat myocardial ischemia/reperfusion injury through activation of PI3K/Akt/Nrf2 pathway. PLoS One 8: e58371, 2013

26. Kern SE, Price-Whelan A and Newman DK: Extraction and measurement of NAD $(\mathrm{P})^{+}$and $\mathrm{NAD}(\mathrm{P}) \mathrm{H}$. Methods Mol Biol 1149: 311-323, 2014.

27. Vessey DA, Li L, Kelley M and Karliner JS: Combined sphingosine, S1P and ischemic postconditioning rescue the heart after protracted ischemia. Biochem Biophys Res Commun 375: 425-429, 2008

28. Meng XY, Yu HL, Zhang WC, Wang TH, Mai X, Liu HT and Xu RC: ZFP580, a novel zinc-finger transcription factor, is involved in cardioprotection of intermittent high-altitude hypoxia against myocardial ischemia-reperfusion injury. PLoS One 9: e94635, 2014

29. Di Lisa F and Ziegler M: Pathophysiological relevance of mitochondria in NAD(+) metabolism. FEBS Lett 492: 4-8, 2001.

30. Eefting F, Rensing B, Wigman J, Pannekoek WJ, Liu WM, Cramer MJ, Lips DJ and Doevendans PA: Role of apoptosis in reperfusion injury. Cardiovasc Res 61: 414-426, 2004

31. Zhao ZQ, Morris CD, Budde JM, Wang NP, Muraki S, Sun HY and Guyton RA: Inhibition of myocardial apoptosis reduces infarct size and improves regional contractile dysfunction during reperfusion. Cardiovasc Res 59: 132-142, 2003.

32. Ling H, Wu L and Li L: Corydalis yanhusuo rhizoma extract reduces infarct size and improves heart function during myocardial ischemia/reperfusion by inhibiting apoptosis in rats. Phytother Res 20: 448-453, 2006.

33. Han Z, Cao J, Song D, Tian L, Chen K, Wang Y, Gao L, Yin Z, Fan $\mathrm{Y}$ and Wang C: Autophagy is involved in the cardioprotection effect of remote limb ischemic postconditioning on myocardial ischemia/reperfusion injury in normal mice, but not diabetic mice. PLoS One 9: e86838, 2014.

34. Cao X, Chen A, Yang P, Song X, Liu Y, Li Z, Wang X, Wang L and Li Y: Alpha-lipoic acid protects cardiomyocytes against hypoxia/reoxygenation injury by inhibiting autophagy. Biochem Biophys Res Commun 441: 935-940, 2013.

35. Balligand JL, Feron O and Dessy C: eNOS activation by physical forces: from short-term regulation of contraction to chronic remodeling of cardiovascular tissues. Physiol Rev 89: 481-534, 2009.

36. Manoury B, Montiel V and Balligand JL: Nitric oxide synthase in post-ischaemic remodelling: new pathways and mechanisms. Cardiovasc Res 94: 304-315, 2012.

37. Jin H, Wang Y, Wang X, Sun Y, Tang C and Du J: Sulfur dioxide preconditioning increases antioxidative capacity in rat with myocardial ischemia reperfusion (I/R) injury. Nitric Oxide 32: 56-61, 2013.

38. Gonzalez FM, Shiva S, Vincent PS, Ringwood LA, Hsu LY, Hon YY, Aletras AH, Cannon RO III, Gladwin MT and Arai AE: Nitrite anion provides potent cytoprotective and antiapoptotic effects as adjunctive therapy to reperfusion for acute myocardial infarction. Circulation 117: 2986-2994, 2008

39. Corr PB and Yamada KA: Selected metabolic alterations in the ischemic heart and their contributions to arrhythmogenesis. Herz 20: 156-168, 1995

40. Doods H and Wu D: Sabiporide reduces ischemia-induced arrhythmias and myocardial infarction and attenuates ERK phosphorylation and iNOS induction in rats. Biomed Res Int 2013: 504320, 2013.

41. Perez NG, Nolly MB, Roldan MC, Villa-Abrille MC, Cingolani E, Portiansky EL, Alvarez BV, Ennis IL and Cingolani HE: Silencing of NHE-1 blunts the slow force response to myocardial stretch. J Appl Physiol 111: 874-880, 2011. 